\title{
The eclipsing millisecond pulsar PSR J1740-5340 and its red straggler companion*
}

\author{
J. A. Orosz ${ }^{\star \star}$ and M. H. van Kerkwijk ${ }^{\star \star \star}$ \\ Astronomical Institute, Utrecht University, PO Box 80 000, 3508 TA Utrecht, The Netherlands
}

Received 7 June 2002 / Accepted 7 October 2002

\begin{abstract}
We present a high-resolution echelle spectrum taken with the Very Large Telescope and analyse archival Hubble Space Telescope photometry of the recently identified companion of the eclipsing millisecond radio pulsar PSR J1740-5340 in the globular cluster NGC 6397. From the spectrum, we show that the companion is metal poor, as expected for a member of NGC 6397. Using synthetic photometry, and assuming a true distance modulus of $12.13 \pm 0.15$ mag and a colour excess of $E_{B-V}=0.179$, we derive a radius of $1.60 \pm 0.17 R_{\odot}$ and an effective temperature of $5410 \pm 50 \mathrm{~K}$, implying a luminosity of $2.0 \pm 0.4 L_{\odot}(1 \sigma$ errors). These properties make it similar to the so-called "sub-subgiants" in the old open cluster M 67 and "red stragglers" in 47 Tuc, which have luminosities comparable to those of turn-off stars, but cooler temperatures and larger radii. The light curve of the companion is well described by ellipsoidal variations, and despite the incomplete $(\sim 60 \%)$ phase coverage, we are able to derive good constraints on a number of the system parameters. In particular, for the inclination we find a $2-\sigma$ lower limit of $i>48^{\circ}\left(i>46^{\circ}\right.$ at $\left.3 \sigma\right)$. Assuming a pulsar mass of $1.2<M_{\mathrm{MSP}}<2.4 M_{\odot}$, this implies a companion mass in the range $0.14<M_{\text {comp }}<0.38 M_{\odot}$. Combined with the photometric constraint, we find a best fit for $i \simeq 50^{\circ}$ and $M_{\text {comp }} \simeq 0.3 M_{\odot}$. We infer a Roche lobe filling factor by radius of $\sim 97 \%$.

Surprisingly, we find no evidence whatsoever for irradiation of the companion, despite the high inferred rotational energy loss of the pulsar $\left(\dot{E} \simeq 1.4 \times 10^{35} \mathrm{erg} \mathrm{s}^{-1}\right.$ ). We discuss possible reasons, but find most lacking. We hypothesise that the system is a triple, and that the acceleration due to a third body in a wide orbit around the binary led to an overestimate of the intrinsic spindown rate and hence the spin-down luminosity. This can be tested by further timing observations. We also discuss two other puzzles, viz., the system's location far outside the cluster core and the companion's large radius and luminosity. We suggest that the system was formed in a binary-binary encounter in the core, due to which the system acquired a substantial velocity, and the companion - which must have been a somewhat evolved turn-off star - lost much of its envelope. We suggest other "red stragglers" and "sub-subgiants" might have formed by similarly drastic encounters.
\end{abstract}

Key words. binaries: close - pulsars: individual: PSR J1740-5340 - globular clusters: individual: NGC 6397

\section{Introduction}

In the course of a survey for pulsars in globular clusters, D'Amico et al. (2001a) discovered an unusual millisecond pulsar (MSP), PSR J1740-5340, in the second-closest globular cluster, NGC 6397. The pulsar has a $3.65 \mathrm{~ms}$ spin period, and is in a circular $\left(e<10^{-4}\right), 1.35-\mathrm{d}$ orbit around a companion

Send offprint requests to: J. Orosz,

e-mail: J.A.0rosz@astro.uu.nl

* Based on observations made with ESO Telescopes at the Paranal Observatories under programme 267.D-5716 and observations made with the NASA/ESA Hubble Space Telescope, obtained from the ST-ECF data archive.

$\star \star$ Present address: Astronomy Dept., San Diego State Univ., 5500 Campanile Drive, San Diego, CA 92182-1221, USA;

e-mail: orosz@sciences.sdsu.edu

$\star \star \star$ Present address: Dept. of Astronomy and Astrophysics, Univ. of Toronto, 60 St George Street, Toronto, ON M5S 3H8, Canada;

e-mail: mhvk@astro.utoronto.ca with a minimum mass of $0.19 M_{\odot}$ (assuming a $1.4 M_{\odot}$ neutron star). In follow-up observations, D'Amico et al. (2001b) found that at a frequency of $1.4 \mathrm{GHz}$, the pulsar signal disappears for about $40 \%$ of the orbit around superior conjunction, and in addition shows irregular intensity and arrival-time variations. By selecting unaffected data sets, D'Amico et al. were nevertheless able to derive a good timing solution, including a precise position, which put the system at 0.55 , or 11 core radii out of the core.

At the timing position, Ferraro et al. (2001) identified a relatively bright $(V \approx 17)$, anomalously red star, which varied at the orbital period. The light curve appeared ellipsoidal, i.e., consistent with what would be expected for a tidally distorted star. This star had been discussed earlier by Taylor et al. (2001), who did not know about the pulsar, but had noted that the companion was a peculiar, variable star with a $\mathrm{H} \alpha-R$ colour indicating weak $\mathrm{H} \alpha$ emission. Taylor et al. also noted that its proper motion was consistent with membership. Finally, 
Grindlay et al. (2001a) identified an X-ray counterpart, with a luminosity of $\sim 8 \times 10^{30} \mathrm{erg} \mathrm{s}^{-1}$.

The system raises many interesting questions, such as:

1. How could the system have gotten so far from the core of the globular cluster? Given their high mass, pulsar systems should quickly settle in the core, as this is indeed generally where they are found. Did the system get kicked out due to a close encounter?

2. How can one understand the strange position of the companion in the colour-magnitude diagram? The star is as luminous as a turn-off star, but redder. As pointed out by Edmonds et al. (2002), these properties are similar to what is seen for the "red stragglers" in 47 Tuc and "subsubgiants" in the old open cluster M 67.

3. What mechanism could cause a wind strong enough to eclipse the pulsar emission? In other eclipsing pulsars, it is thought the pulsar irradiation drives a wind, but the light curve for PSR J1740-5340 shows no indication for heating. Could the wind be intrinsic?

Fortunately, the companion is bright, and hence detailed studies are possible. This opens the possibility not only of addressing the above questions, but also of measuring the system parameters accurately. The latter would be particularly interesting since no accurate masses exist for MSPs. As MSPs are expected to be more massive due to accretion, this might be interesting also from the point of view of constraining the equation of state of ultra-dense matter (for a review, see Lattimer \& Prakash 2001): neutron stars with masses above $\sim 1.6 M_{\odot}$ cannot exist for so-called soft equations of state, in which matter at high densities is relatively compressible (e.g., due to meson condensation or a transition between the hadron and quarkgluon phases). Obviously, if one were to have solid evidence that massive neutron stars exist, these equations of state would be falsified.

Given the above, a thorough observing programme seems justified. In this paper, we report on the analysis of archive photometry and present the results of a pilot programme designed to test the feasibility of high resolution spectroscopic observations.

\section{Observations}

\subsection{Photometry}

The photometric data we used were taken from the public $H S T$ archive. The observations were carried out on 1996 March in the F336W, F439W, F555W, and F814W filters (hereafter $U_{336}$, $B_{439}, V_{555}$, and $\left.I_{814}\right)$ and 1999 April using the $V_{555}, \mathrm{~F} 675 \mathrm{~W}$ (hereafter $R_{675}$ ), $I_{814}$, and $\mathrm{F} 656 \mathrm{~N}$ (hereafter $\mathrm{H} \alpha_{656}$ ) filters. Light curves of the companion were constructed using the HSTphot package (version 1.1; Dolphin 2000).

In Fig. 1, we show the folded light curves in all bands. As discussed by Ferraro et al. (2001), the overall shape of the lightcureve is reminiscent of ellipsoidal variations, in which the maxima are at the orbital quadratures, when the tidally distorted companion is seen from the side. We find that the curves in $U_{336}, B_{439}, R_{675}$, and $\mathrm{H} \alpha_{656}$ curves are reasonably

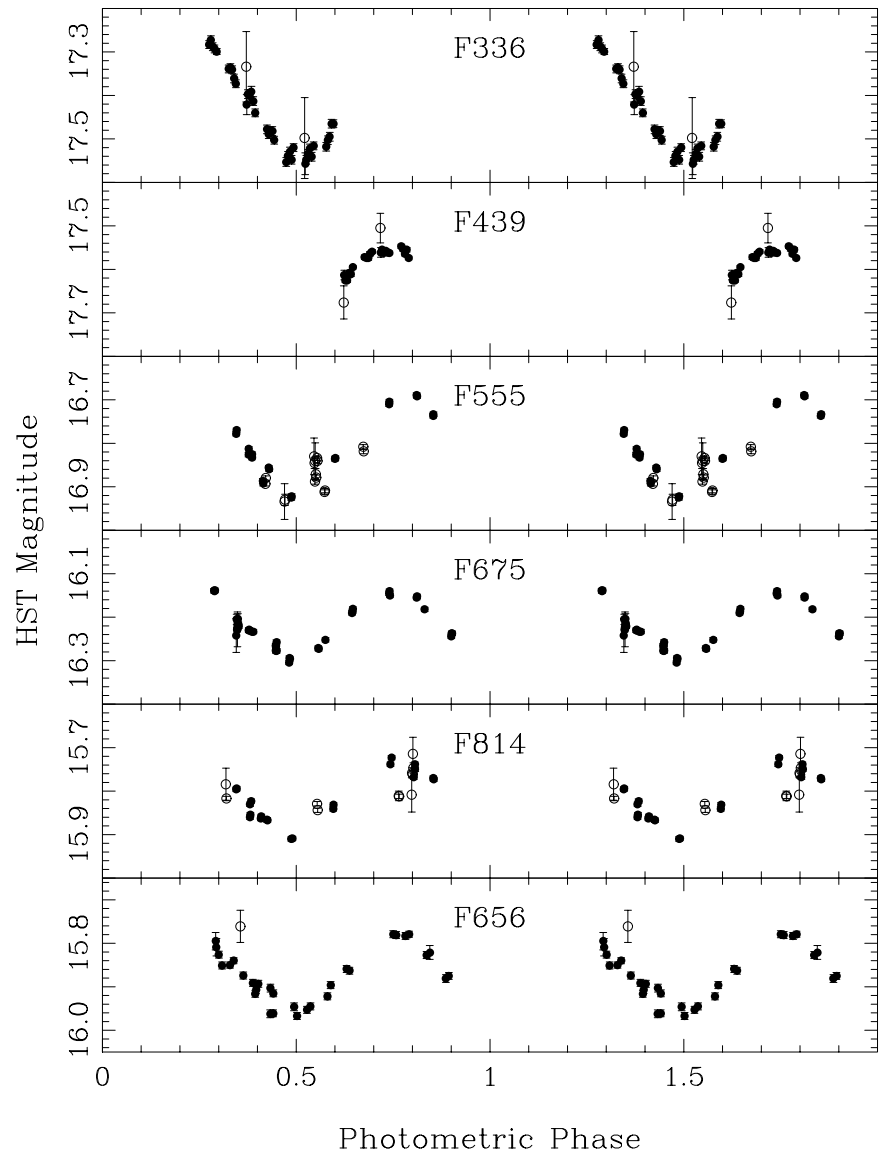

Fig. 1. The HST data as a function of photometric phase. Phase zero is the time of the inferior conjunction of the companion star. The points plotted with the open circles are excluded from analysis.

smooth and show the ellipsoidal modulations quite nicely, but that those in $V_{555}$ and $I_{814}$ have some deviant points. We believe the latter are due to the very close proximity of a bad column on the CCD. Indeed, the $R_{675}$ and $\mathrm{H} \alpha_{656}$ band data from MJD 51273 show smooth variations whereas the $V_{555}$ and $I_{814}$ data from that same day both show deviant points. Also, the $V_{555}$ and $I_{814}$ light curves are the only ones with data from both 1996 and 1999. Removing the 1996 data improves the $I_{814}$ band light curve somewhat but does not really improve the $V_{555}$ light curve.

The photometric data that we will use in our analysis below are shown as the solid points in Fig. 1. The excluded $V_{555}$ data are those from 1996 plus eight points from MJD $51272.807-51272.819$. The excluded $I_{814}$ data are those from 1996 plus five points from MJD 51 271.794-51 271.800 and two points from near MJD 51272.818 . Also, two points in $U_{336}$, two points in $B_{439}$, and one point in $\mathrm{H} \alpha_{656}$ were excluded owing to the large uncertainties.

As is clear from Fig. 1, the phase coverage of the HST light curves is not complete, and clearly additional photometry would be desirable. Since the field of the pulsar is relatively crowded, we present in Fig. 2 a finding chart as an aid for ground-based observers. This image was obtained May 14, 1999 with the Wide Field Imager on the $2.2 \mathrm{~m}$ telescope at ESO, La Silla. 


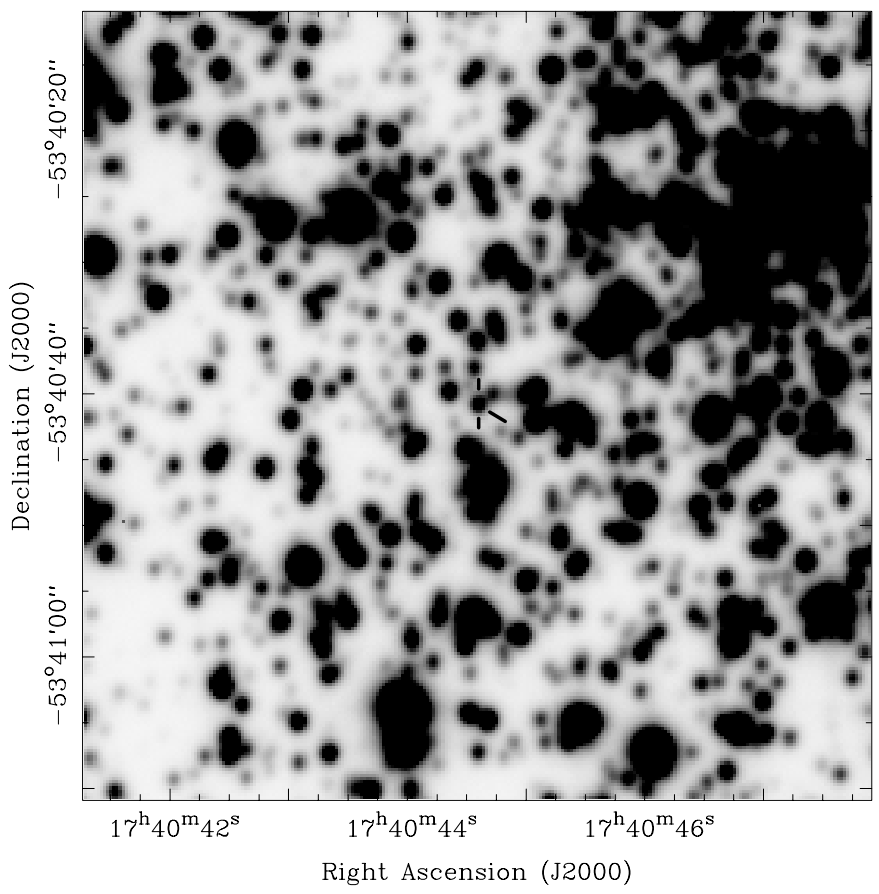

Fig. 2. Finding chart for PSR J1740-5340. This image is a $1 \times 1$ arcmin subsection of a $V$-band image obtained May 14, 1999 with the Wide Field Imager on the ESO $2.2 \mathrm{~m}$ telescope at La Silla.

\subsection{Spectroscopy}

A 2880-second UVES spectrum in 0.8 arcsecond seeing was obtained for us in Director's Discretionary Time on 2001 September 20, starting at 23:56 UT. UVES resides at the Nasmyth focus of Kueyen, the second of the four 8.2-m Very Large Telescopes at Paranal (Dekker et al. 2000; D'Odorico et al. 2000). UVES is a double-arm instrument, with a $2048 \times 4096$ thinned, antireflection-coated CCD (EEV CCD$44,15 \mu \mathrm{m}$ pixels) in the blue arm and a mosaic of two $2048 \times 4096$ CCDs (EEV CCD-44 and MIT/LL CCD-20, both with $15 \mu \mathrm{m}$ pixels) in the red arm. In this paper we will only make use of the data from the red arm. The standard "DIC1 346+580" configuration was used, which gives a wavelength coverage of 4765-6830 $\AA$ in the red arm. With a 1 " wide slit, the resolving power is about 40000 . The CCDs were read out in the $2 \times 2$ pixel binned mode. We used IRAF to calibrate the data and extract the spectra. The typical signal-to-noise ratio is about 20 per resolution element.

\section{Analysis}

\subsection{Radius and temperature}

We can use the fact that PSR J1740-5340 is a member of the globular cluster NGC 6397 (see also Sect. 3.2) to measure the radius and effective temperature of the companion. For this exercise we use synthetic photometry for the HST filters derived from the NextGen models (Hauschildt et al. 1997; F. Allard 2001, private communication) with a metallicity of $[\mathrm{Fe} / \mathrm{H}]=-2$, appropriate for NGC 6397 (e.g., Castilho et al. 2000; Thévenin et al. 2001). The synthetic photometry allows us to compute the expected absolute magnitude of a star in a

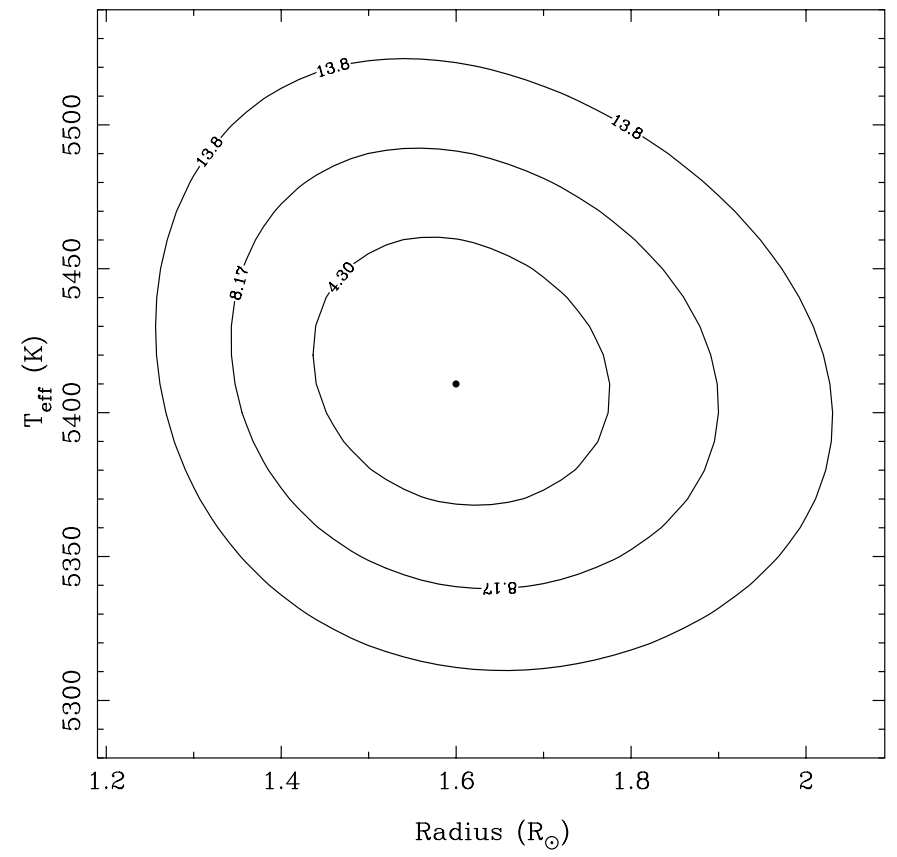

Fig. 3. The radius and temperature of the companion star derived by comparing the HST magnitudes and colours with synthetic photometry from the NextGen model atmospheres (Hauschildt et al. 1997; F. Allard 2001, private communication). The contours show the 1, 2, and $3 \sigma$ confidence limits for two parameters of interest $\left(\Delta \chi^{2}=4.30\right.$, 8.17, and 13.8, respectively). We find $R_{\text {comp }}=1.60 \pm 0.17 R_{\odot}$ and $T_{\text {eff }}=$ $5410 \pm 50 \mathrm{~K}$, assuming a reddening of $E_{B-V}=0.179$ (Anthony-Twarog $\&$ Twarog 2000) and a true distance modulus of $12.13 \pm 0.15$ mag (Reid $\&$ Gizis 1998; see text for a discussion on the distance).

given $H S T$ bandpass and various colours as a function of its effective temperature $T_{\text {eff }}$, surface gravity $\log g$, and radius $R_{\text {comp }}$.

To compare the synthetic magnitudes with the observations, we need to determine the "mean" apparent magnitudes. For this purpose, we used the ELC code (Orosz \& Hauschildt 2000), as follows. First, we fit an ellipsoidal model (see Sect. 3.3) to the light curve in the $R_{675}$ filter (which has the most complete phase coverage). We then computed the flux of a spherical star with the same effective temperature and effective radius and applied the same scaling as the fitted ellipsoidal model. The spherical star would have an $R_{675}$ magnitude of 16.25 .

To convert the above apparent magnitude to an absolute one, we need to know the reddening and the distance. For the reddening, we adopt $E_{B-V}=0.179 \pm 0.003$, as determined by Anthony-Twarog \& Twarog (2000) from $u v b y \mathrm{H} \beta$ photometry, and use the interpolated extinction curve for the WFPC2 filters appropriate for a K5 spectral type (Holtzman et al. 1995). For the distance, we use a distance modulus $(m-M)_{0}=$ $12.13 \pm 0.15$, as inferred by Reid \& Gizis (1998) from mainsequence fitting to local metal-poor $\mathrm{M}$ subdwarfs with welldetermined parallaxes.

We note that the uncertainty in the distance dominates, not only because of the large random error, but also because of possible systematic biases. Indeed, from different methods rather different values are found. In an earlier analysis based on subdwarf G and F stars, Reid (1998) found a distance modulus of $12.24 \pm 0.10$, i.e., a larger distance. In contrast, Harris (1996) 
finds a smaller distance -11.80 with an estimated uncertainy of 0.1-0.2 mag - based on the apparent magnitude of the horizontal branch. Finally, Gratton et al. (2002) present preliminary results from a more advanced main-sequence fit, based on precise matching of spectral types with high-resolution spectroscopy, and $E_{B-V}=0.183 \pm 0.005,[\mathrm{Fe} / \mathrm{H}]=-2.03 \pm 0.04$, and $(m-M)_{0}=12.01 \pm 0.06$. For our analysis, we use the Reid \& Gizis (1998) distance, since this seems the most reliable of the published results, and is the one used by most authors so far. However, where appropriate, we will mention explicitly the implications of assuming the shorter Gratton et al. (2002) distance.

With our adopted reddening and distance modulus, we find an absolute magnitude in the F675W bandpass of $M_{675}=$ $(16.25-0.44-12.13) \pm 0.15=3.64 \pm 0.15$, where the error in the absolute magnitude was taken to be the error in the distance modulus. A similar procedure was used to find the colours; we found $B_{439}-V_{555}=0.867, V_{555}-R_{675}=0.597$, and $R_{675}-I_{814}=0.406$.

For a given combination of $T_{\text {eff }}, \log g$, and $R_{\text {comp }}$, we can form a $\chi^{2}$ :

$$
\begin{aligned}
\chi^{2}= & \frac{\left[(B-V)_{\mathrm{syn}}-0.867\right]^{2}}{\sigma_{\text {colour }}^{2}}+\frac{\left[(V-R)_{\mathrm{syn}}-0.597\right]^{2}}{\sigma_{\text {colour }}^{2}} \\
& +\frac{\left[(R-I)_{\mathrm{syn}}-0.406\right]^{2}}{\sigma_{\text {colour }}^{2}}+\frac{\left[M_{675, \mathrm{syn}}-3.64\right]^{2}}{0.15^{2}} .
\end{aligned}
$$

Here, $\sigma_{\text {colour }}$ is the uncertainty in the colours; we adopt $\sigma_{\text {colour }}=0.0144$, which gives $\chi_{v}^{2}=1$ at the minimum. We found that the magnitude and colours hardly depend on gravity, so we fixed $\log g$ at its nominally best value of 3.7. This leaves the effective temperature $T_{\text {eff }}$ and radius $R_{\text {comp }}$ as the two free parameters. Figure 3 shows the contours of $\chi^{2}$ in the $R_{\text {comp }}-T_{\text {eff }}$ plane. We find $R_{\text {comp }}=1.60 \pm 0.17 R_{\odot}$ and $T_{\text {eff }}=5410 \pm 50 \mathrm{~K}$. These values are consistent with what Ferraro et al. (2001) found by independent means. Assuming the Gratton et al. (2002) distance modulus, the inferred radius and temperature would be $1.52 \pm 0.08 R_{\odot}$ and $5420 \pm 40 \mathrm{~K}$, respectively.

\subsection{Radial and rotational velocities, and metallicity}

In Fig. 4, we show the spectrum of PSR J1740-5340 near the $\mathrm{Mg} \mathrm{b}$ lines. The lines are clearly broadened, as expected if the star is co-rotating with the $1.35-\mathrm{d}$ orbit.

We measured the radial and rotational velocities of the companion star using a template, for which we chose the metal poor star HD $122196\left(V=8.73, E_{B-V}=0.01\right)$. This star has an effective temperature and gravity $\left(T_{\text {eff }}=5850 \pm 100 \mathrm{~K}\right.$, $\log g=3.5$; Ryan \& Deliyannis 1998) quite similar to what we found for the companion star of PSR J1740-5340, as well as a metallicity $([\mathrm{Fe} / \mathrm{H}]=-1.93 \pm 0.10$; ibid. $)$ similar to that of NGC 6397 members. Furthermore, this star was observed with UVES using the same instrumental configuration as what was used for PSR J1740-5340, although at a slightly higher resolving power $(R=50000)$.

To measure the radial velocity by cross correlation, we have to iterate a bit, since we have to rotationally broaden

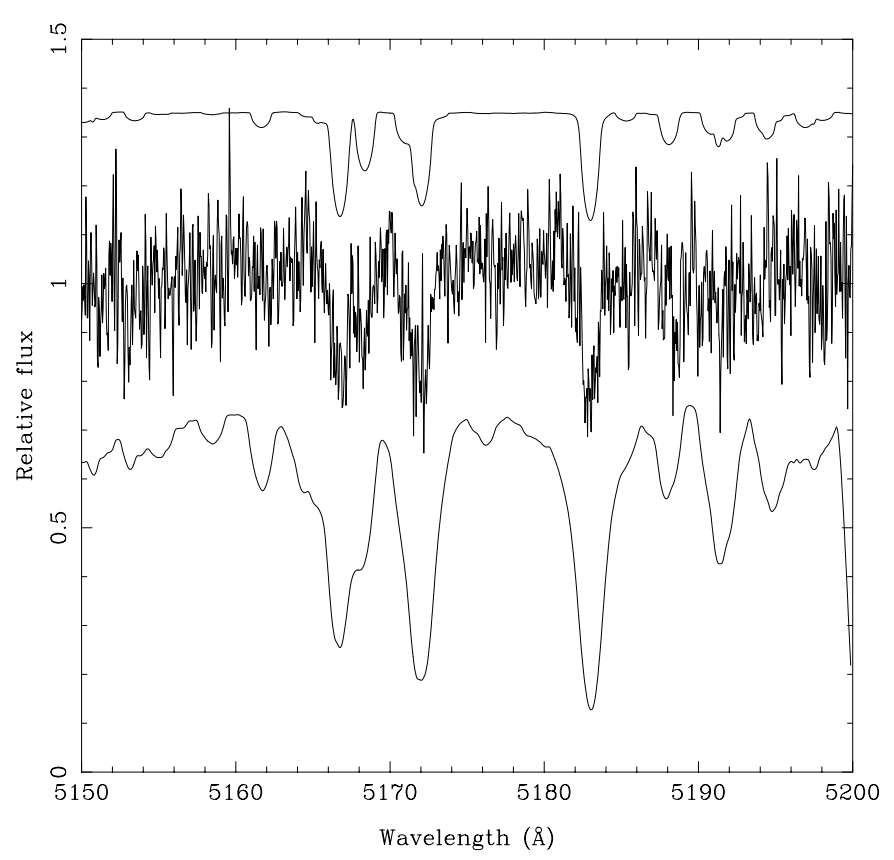

Fig. 4. UVES spectra of the companion of PSR J1740-5340 (middle), compared with spectra broadened to $v_{\text {rot }} \sin i=52 \mathrm{~km} \mathrm{~s}^{-1}$ of the metalpoor star HD 122196 (top; $T_{\text {eff }}=5850 \pm 100 \mathrm{~K}, \log g=3.5,[\mathrm{Fe} / \mathrm{H}]=$ $-1.93 \pm 0.10$; Ryan \& Deliyannis 1998), and of the solar-metallicity star HR 996 (bottom; $T_{\text {eff }}=5667 \mathrm{~K}, \log g=4.29,[\mathrm{Fe} / \mathrm{H}]=-0.01$; Pasquini et al. 1994, taken from the atlas of Montes \& Martin 1998).

the template to match the rotational velocity of the companion. Given a radius of $R_{\text {comp }}=1.60 R_{\odot}$ and assuming synchronous rotation, one expects $v_{\text {rot }} \sin i=42.3 \mathrm{~km} \mathrm{~s}^{-1}$ for an inclination of $45^{\circ}$ and $59.8 \mathrm{~km} \mathrm{~s}^{-1}$ for an inclination of $90^{\circ}$. We initially broadened the spectrum of HD 122196 (which has negligible intrinsic rotational broadening) by $49 \mathrm{~km} \mathrm{~s}^{-1}$ and measured the radial velocities of each PSR J1740-5340 echelle order with the IRAF task fxcor, an implementation of the Tonry \& Davis (1979) cross correlation technique. Good cross correlation peaks were found for 14 echelle orders, and the weighted average of the (heliocentric) velocities from each order is $135 \pm 1 \mathrm{~km} \mathrm{~s}^{-1}$ (assuming HD 122196 has a heliocentric velocity of $-23 \mathrm{~km} \mathrm{~s}^{-1}$ ).

To find the rotational velocity of the companion, the spectrum of HD 122196 was Doppler shifted to remove the relative velocity difference between it and the PSR J1740-5340 spectrum, and the $\chi^{2}$-based matching technique of Marsh et al. (1994) was used to measure the projected rotational velocity. Using the spectral region near the $\mathrm{Mg} b$ lines, which contains the strongest lines, we find $v_{\text {rot }} \sin i=52 \pm 4 \mathrm{~km} \mathrm{~s}^{-1}$. This value should be treated with caution owing to the relatively low signal-to-noise and the fact that the template spectrum may not be a perfect match (e.g., owing to differences in the $\mathrm{Mg}$ and/or Fe abundances). We re-measured the radial-velocity measurement using this new value of $v_{\text {rot }} \sin i$, but found that it did not change the result.

In Fig. 4, we compare the spectrum of PSR J1740-5340 and the spectrum of HD 122196, broadened using $V_{\text {rot }} \sin i=$ $52 \mathrm{~km} \mathrm{~s}^{-1}$. To the extent one can judge, given the rather noisy spectrum, the match is reasonable. For comparison, we also 
show a high resolution spectrum of HR 996, a star with a similar temperature as PSR J1740-5340, but with solar metallicity. The Mg b lines in HR 996 are clearly much stronger than they are in PSR J1740-5340. Given this and the reasonable match between PSR J1740-5340 and the metal poor star HD 122196, we conclude that the companion in PSR J1740-5340 is metal poor, providing further confirmation that it is a member of the globular cluster NGC 6397 and not a foreground object in the Galactic disc.

Assuming the MSP is bound to the cluster, our single radial velocity measurement allows us to make a rough estimate of the radial velocity amplitude $K_{\text {comp }}$. This is because the cluster membership constrains the systemic velocity $\gamma$, and thus we can calculate $K_{\text {comp }}$ from $K_{\text {comp }}=\left(v_{\text {obs }}-\gamma\right) / \sin (2 \pi \phi)$, where $\phi=0.368$ is the phase relative to inferior conjunction of the companion. Given the radial velocity of NGC 6397 of $18.9 \pm 0.1 \mathrm{~km} \mathrm{~s}^{-1}$ (Harris 1996) and its escape velocity of $\sim 19 \mathrm{~km} \mathrm{~s}^{-1}$ (Webbink 1984), we take $0<\gamma<38 \mathrm{~km} \mathrm{~s}^{-1}$, and infer $132<K_{\text {comp }}<183 \mathrm{~km} \mathrm{~s}^{-1}$. The pulsar timing yields $K_{\mathrm{MSP}}=26.612 \mathrm{~km} \mathrm{~s}^{-1}$ (D'Amico et al. 2001b), and hence the mass ratio $Q \equiv M_{\mathrm{MSP}} / M_{\text {comp }}$ should be in the range $5<Q<7$. Obviously, one should not rely on a single radial velocity point to measure the amplitude of the companion star's velocity curve. Nevertheless, this exercise at least gives a rough idea of what the mass ratio might be. As we will see below, the values we find are consistent with what we infer from the other data on the system.

\subsection{Ellipsoidal modelling}

The HST lightcurves of PSR J1740-5340 are, as mentioned above, reminiscent of ellipsoidal modulation, with maxima at the quadratures, and minima at the conjunctions. Below, we will use models for a tidally distorted star to reproduce the lightcurve and to estimate some of the system parameters. Before doing so, we should caution that the phase coverage is incomplete, and that, in particular, superior conjunction of the pulsar is missing. Thus, even though we obtain good fits to the data at hand, whether or not our models are appropriate, remains to be fully verified.

For our modeling of the HST light curves, we used the ELC code (Orosz \& Hauschildt 2000). We have three unknown geometrical parameters: the mass ratio $Q$, the inclination $i$, and the Roche lobe filling factor by radius $f$. Usually, one also has the orbital separation $a$ as an unknown. For PSR J1740-5340, however, the projected semi-major axis of the MSPs orbit is known extremely accurately ( $x \equiv$ $a_{\mathrm{MSP}} \sin i / c=1.65284 \pm 0.00007 \mathrm{lt}-\mathrm{s}$; the $1 \sigma$ error corresponds to about $7 \mathrm{~km}$; D'Amico et al. 2001b). As a result, we can write the orbital separation as a function of the inclination and the mass ratio, $a=c(Q+1) x / \sin i$.

We also require parameters related to the companion. These, however, can be fixed at reasonable values. We assume the companion star is rotating synchronously with the orbit, fix the mean temperature at the value determined above, and fix its gravity darkening exponent at $\beta=0.1$ (Claret 2000). We used specific intensities for models with $[\mathrm{Fe} / \mathrm{H}]=-2$ from Kurucz ${ }^{1}$ (1979), integrated with the WFPC2 filter response curves. The use of the tabulated specific intensities removes the need for a parameterised limb darkening law.

Finally, we need to consider irradiation by the pulsar. As we will discuss at length in Sect. 4, this should have had a dominant effect on the light curve, but no effect whatsoever is seen. For the remainder of this section, therefore, we will ignore it.

In any fitting procedure, one must assign weights to the observations. We do this by fitting an ellipsoidal model to each bandpass separately, and scaling the error bars of the data points in that bandpass so that the reduced $\chi^{2}$ of the fit equals 1 at the minimum. The scaled errors are typically between about 0.015 and $0.022 \mathrm{mag}$, which is on the order of the claimed accuracy of HSTphot (Dolphin 2000), and thus provides some evidence that our model is appropriate. Next, using these scaled errors, we fit all of the bandpasses simultaneously. With this procedure, somewhat reduced weight is given to bands in which the light curves are relatively ratty, such as $V_{555}$ and $I_{814}$. For our best fits, we find $\chi^{2} \simeq 225$ for 172 data points in the 6 filters, i.e., a reduced $\chi^{2}$ which is still reasonably close to unity. This shows that best fits to the lightcurves in the different bands are mutually consistent.

For our fits, we found it convenient to define a grid of points in the mass ratio-inclination plane and to make the grid spacing in the inclination axis equal in $\sin i$ rather than $i$. At each point in this plane, the values of the mass ratio and the sine of the inclination are fixed according to the location in the plane, leaving the Roche lobe filling factor $f$ as the only free fitting parameter. In Fig. 5, we show the results of the ellipsoidal fitting as the dashed contours. We find that good fits are possible over a large range of inclinations: the formal $2 \sigma$ range includes all $i>49.5^{\circ}(\sin i>0.76)$. The mass ratio $Q$ is not constrained.

In Fig. 5d, solid contours show the variation of the bestfitting filling factor in the $Q-\sin i$ plane (here, the filling fraction is defined as $R_{\text {comp }} / R_{\text {Roche }}$ ). From these, the reason for the large allowed range in inclination becomes clear: the amplitude of the ellipsoidal variations can be matched by stars viewed almost edge-on that underfill the Roche lobe significantly and hence are less strongly distorted, as well as by stars viewed more inclined that almost fill the Roche lobe and thus are maximally distorted.

The similarity in the fits can be see in Fig. 6, which shows some representative ellipsoidal fits overdrawn on the folded HST light curves. The first model is very near the formal $\chi^{2}$ minimum, with $i=60^{\circ} 5, Q=6$, and a filling fraction by radius of $91.1 \%$ for the companion star. The second model is also for $Q=6$, but for a Roche-lobe filling companion. In this case, $i=46^{\circ} .7$. Although the $\chi^{2}$ values are somewhat different $\left(\chi^{2}=225\right.$ and $\chi^{2}=236$, respectively), the quality of the fits looks very similar to the eye.

Turning back to Fig. 5d, we see that because a star cannot become larger than the Roche lobe, the fits get worse very quickly for inclinations less than $49^{\circ}$ : the $3 \sigma$ contour is at $i \approx 46^{\circ}(\sin i \approx 0.72)$ near $Q=6$ and the $99.99 \%$ confidence limit is at $i \approx 45^{\circ}(\sin i \approx 0.71)$. At $i \approx 40^{\circ}(\sin i \approx 0.64$; the

\footnotetext{
${ }^{1}$ http://www. cfaku5.harvard.edu
} 

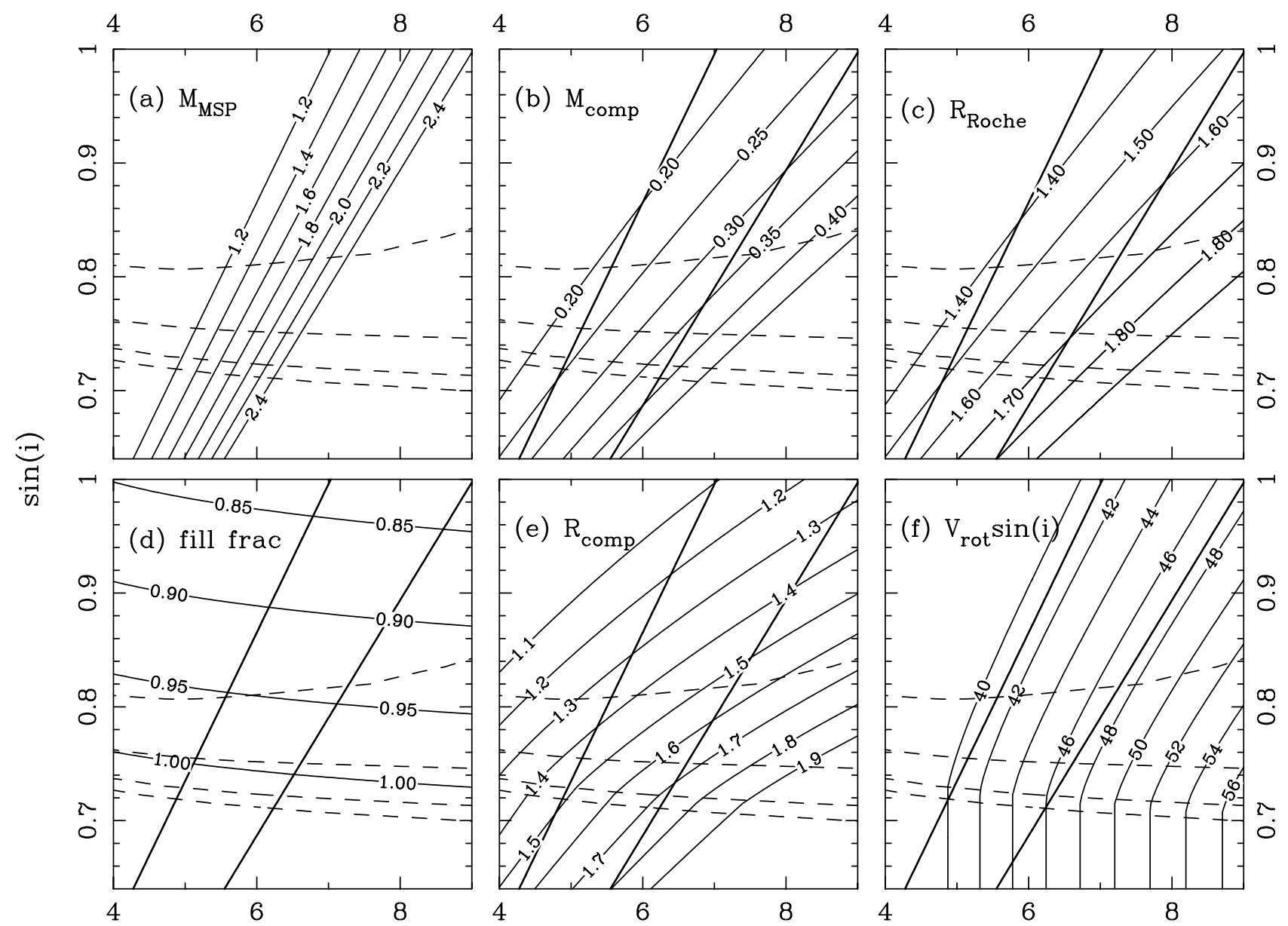

Mass Ratio

Fig. 5. Constraints on the system parameters in the mass-ratio, inclination plane. In all panels, the dashed lines indicate, from top to bottom, the $1 \sigma, 2 \sigma, 3 \sigma$, and $99.99 \%$ confidence limits inferred from our ellipsoidal fits to the six HST light curves. The fits set a stringent lower limit to the inclination, but hardly constrain the mass ratio. a) Contours of the MSP mass (in solar masses). For reasonable neutron star masses $\left(1.2 M_{\odot}-2.4 M_{\odot}\right)$ a relatively small part of the plane is filled. In all other panels, the thick diagonal lines denote the borders of the region where $1.2 \leq M_{\mathrm{MSP}} \leq 2.4 M_{\odot}$. b) Contours of the companion star mass (in solar masses). c) Contours of constant Roche-lobe radius of the companion (in solar units). d) Contours of constant best-fitting filling fraction of the companion star (by radius, i.e., $R_{\text {comp }} / R_{\text {Roche }}$ ). e) Contours of the constant companion star radius corresponding to the best-fitting filling factor (in solar units). f) Contours of constant projected rotational velocity corresponding to the best-fitting companion radius (in $\mathrm{km} \mathrm{s}^{-1}$ ).

bottom of all panels) the ellipsoidal fits are hopelessly bad, as can be seen in Fig. 6: the amplitudes are much too small. Thus, we regard $i>40^{\circ}$ as a very firm lower limit.

In the other panels of Fig. 5, contours are drawn for various other quantities. The top row shows the MSP mass (Fig. 5a), the companion star mass (Fig. 5b), and the Roche lobe radius of the companion (Fig. 5c); these quantities can be calculated from $Q$ and $\sin i$ directly, and the contours are thus independent of the fit. If we use the limit on the inclination, and restrict ourselves to a reasonable range for the mass of the neutron star $\left(1.2 M_{\odot}<M_{\mathrm{MSP}}<2.4 M_{\odot}\right)$, then we see from panel a that we are left with only a relatively small part of the parameter space: the mass ratio is in the range $5 \lesssim Q \lesssim 9$ for $i \geq 45^{\circ}$. Given this region where the neutron star mass is reasonable, we see in Fig. 5b that the mass of the companion star is in the range $0.14 M_{\odot}<M_{\text {comp }}<0.38 M_{\odot}$. From Fig. 5c, the Roche radius of the companion is smaller than the radius derived from
$H S T$ photometry for inclinations near $90^{\circ}$ unless the mass ratio $Q$ is very large.

In the bottom row, quantities are shown that do depend on the light curve fit. Figure 5e shows the best-fitting radius for the companion star; this is simply the best-fitting filling fraction times the Roche lobe radius. We see that for high inclinations ( $\sin i \geq 0.9$ or $i>64^{\circ}$ ) the fitted radius of the companion star is much smaller than the radius derived from the HST photometry. On the other hand, the fitted radius is comparable to the radius derived from the $H S T$ photometry for $i \approx 50^{\circ}(\sin i \approx 0.77$ ) and $Q \approx 6$. This is also consistent with the rough estimate of $Q$ from our single radial velocity.

Finally, Fig. 5f shows contours of the best-fitting projected rotational velocity of the companion star (i.e., proportional to best-fitting radius times $\sin i$ ). The contours bend where the star starts to fill its Roche lobe. We note that our present value of $v_{\text {rot }} \sin i=52 \mathrm{~km} \mathrm{~s}^{-1}$ is well outside the range of parameter 


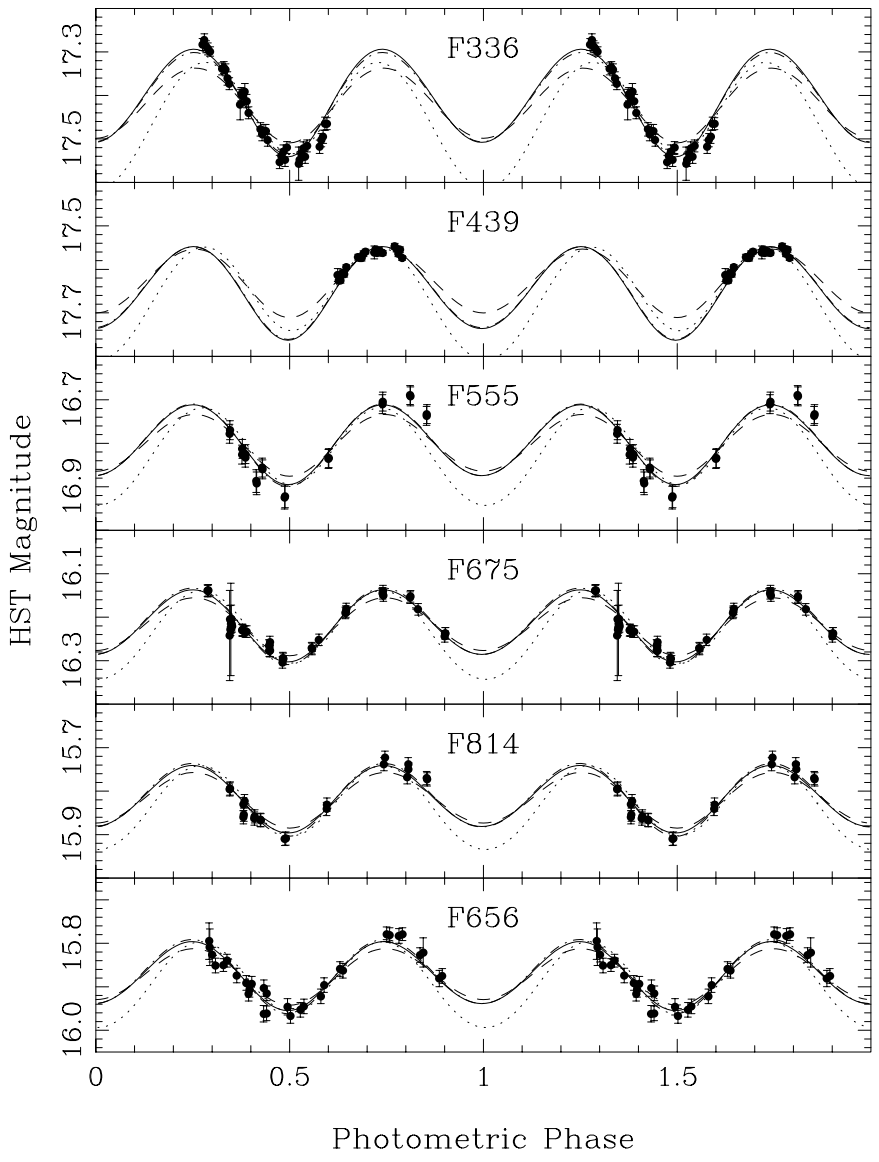

Fig. 6. Ellipsoidal models with no X-ray heating overdrawn on the $H S T$ light curves. The solid lines are for a model with $i=60^{\circ} 5, Q=6$, and a filling fraction by radius of $91.1 \%$ for the companion star. The fit has $\chi^{2}=225$ and is near the minimum. The dash-dotted lines denote a model with a Roche lobe filling companion, $i=46.7^{\circ}$, and $Q=6$. The fit has $\chi^{2}=236$ and is at the formal $2 \sigma$ limit. The dashed lines denote a model with a Roche lobe filling companion, $i=40^{\circ}$, and $Q=6$. The fit has $\chi^{2}=411$; it is clearly excluded by the data. Finally, the dotted line denotes a model with a Roche lobe filling companion, $i=52^{\circ} .3$, $Q=6$, and an albedo of $10 \%$. The fit has $\chi^{2}=411$, and is also clearly excluded by the data.

space where the mass of the MSP is reasonable. However, the statistical error on $v_{\text {rot }} \sin i$ is large (e.g., a $2 \sigma$ deviation down to $V_{\text {rot }} \sin i=44 \mathrm{~km} \mathrm{~s}^{-1}$ would give a reasonable mass for the MSP) and, as mentioned, there may be systematic errors as well. The uncertainties associated with the ellipsoidal modelling are smaller: the $1-\sigma$ uncertainty in the actual predicted rotational velocity of the contours in Fig. $5 \mathrm{f}$ is about $0.1 \mathrm{~km} \mathrm{~s}^{-1}$.

\section{The puzzling lack of heating}

We found that the light curve displayed by the companion is described well by ellipsoidal modulation. This is remarkable, as we would have expected to see strong heating: the pulsar's rotational energy loss, $L_{\mathrm{sd}}=I \Omega \dot{\Omega}=1.4 \times 10^{35} I_{45} \mathrm{erg} \mathrm{s}^{-1}$ (D'Amico et al. $2001 \mathrm{~b}$ ) is roughly 19 times the bolometric luminosity of the companion. We calculated models in which we included the effect of heating, assuming isotropic emission. If we assume that the companion has an albedo or "reprocessing efficiency" with the canonical value of 0.5 , then the expected $V_{555}$ light curve would be nearly sinusoidal with an amplitude of 0.4 mag and would have a maximum at phase 0.5 , rather than the minimum that is observed. Even an albedo of only $10 \%$ leads to a horrible fit, as can be seen in Fig. 6. Indeed, if we leave the albedo as a free parameter, we find that it must be $1 \%$ or less in order to match the observed light curves; the best-fitting value is zero.

The limit of $1 \%$ is in striking contrast to what is seen in all pulsar systems in which irradiation could be observed. In particular, for the so-called "black-widow" pulsars, which show light curves dominated by heating, the inferred reprocessing efficiencies are $\sim 10 \%$ for PSR B1957+20 (Callanan et al. 1995) and $\sim 40 \%$ for PSR J2051-0827 (Stappers et al. 2001). A high efficiency, of $\sim 60 \%$, has also been inferred for the MSP 47 Tuc U and its low-mass white dwarf companion (Edmonds et al. 2001), and strong heating is observed for the pulsar, main-sequence star binary 47 Tuc W (Edmonds et al. 2002).

What could be the cause of this apparent low efficiency? The following seem possible: (i) the pulsar's luminosity is lower than inferred from its spin period and period derivative; (ii) the pulsar radiation is emitted non-isotropically, largely missing the companion; (iii) the pulsar radiation is absorbed or deflected on the way to the companion; (iv) the radiation is absorbed sufficiently deep that it can be transported, e.g., by winds as in Jupiter, and re-emitted isotropically.

On empirical grounds, we believe we can exclude possibilities (iii) and (iv): no evidence is seen for either in the above-mentioned systems, which include companions ranging from brown dwarfs to main-sequence stars and white dwarfs. Also anisotropy seems excluded: while pulsars winds are far from isotropic, as is clear from the Crab and its nebula (e.g., Weisskopf et al. 2000), it is hard to see how a companion could fail to be irradiated over its whole orbit. Indeed, the smooth light curves of the black widow pulsars and symmetric bow shocks around the MSP binaries PSR B1957+20 (Kulkarni \& Hester 1988) and PSR J0437-4715 (Bell et al. 1995) argue against the required extreme anisotropies.

If the above reasoning is correct, we are left with the possibility of a spin-down luminosity lower than inferred from the spin period and its derivative. This could happen if the spin period derivative does not reflect actual spin-down, but rather an (apparent) acceleration, of order $a / c=\dot{P} / P=$ $4.6 \times 10^{-17} \mathrm{~s}^{-1}$. Expected contributions to $\dot{P}$ arise from proper motion, acceleration in the cluster and differential galactic acceleration between the cluster and us. Using estimates from Phinney (1992), we find that the Galactic acceleration is far too small, $\sim 1.6 \times 10^{-19} \mathrm{~s}^{-1}$, while the required proper motion, $(c \dot{P} / P d)^{1 / 2} \simeq 85 \mathrm{mas} \mathrm{yr}^{-1}$ or $\sim 1000 \mathrm{~km} \mathrm{~s}^{-1}$ at the distance of NGC 6397, is outrageously high (and excluded by the HST observations).

The maximum acceleration in a cluster is approximately $1.1 G \Sigma_{M} / c$ (Phinney 1992), where $\Sigma_{M}$ is the surface density enclosed within the projected distance of the pulsar from the core. With an extinction-corrected $V$-band surface brightness within 0.55 of $\Sigma_{V, 0}=18.0 \mathrm{mag} \operatorname{arcsec}^{-2}$ (Trager et al. 1995), and a mass to light ratio of about 3 (in solar units), we find 
a maximum acceleration of $\sim 3 \times 10^{-18} \mathrm{~s}^{-1}$, again far short of that required (as found by D'Amico et al. 2001b using a different method). We note that the mass to light ratio is uncertain; for instance, from negative period derivatives of two pulsars in NGC 6752, D' Amico et al. (2002) infer a mass to light ratio of $\gtrsim 10$. In order for the acceleration to match the period derivative for PSR J1740-5340, however, a mass to light ratio of $\sim 50$ would be required. This seems excessive.

\subsection{A third body?}

Having excluded all likely causes, what remains, however improbable? One possibility is that the binary is passing another star close by or that it is part of a triple system, and happens to be seen being accelerated away from us. This would not be unprecedented: PSR B1620-26 in M4 has a $\sim 0.3 M_{\odot}$ white-dwarf companion in a $191 \mathrm{~d}$ orbit, as well as a $\sim 0.007 M_{\odot}$ giant planet or brown dwarf like object in a $\sim 300 \mathrm{yr}, e \simeq 0.45$ orbit (Ford et al. 2000). For PSR J1740-5340, a third object with mass $M_{3}$ at separation $a_{3}=\left(f G M_{3} P / \dot{P} c\right)^{1 / 2}=200\left(f M_{3} / 0.1 M_{\odot}\right)^{1 / 2} \mathrm{AU}$ would be required; here $f=\sin i_{3} \sin \phi_{3}$ with $i_{3}$ the inclination and $\phi_{3}$ the orbital phase relative to the ascending node (a positive acceleration the third object to be behind the pulsar binary, or $\sin \phi_{3}>0$ ). The corresponding period (or timescale in case of a close encounter) is $P_{3}=2 \pi\left(a^{3} / G M\right)^{1 / 2} \simeq$ $400\left(f M_{3} / 0.1 M_{\odot}\right)^{3 / 2}\left(M / 1.7 M_{\odot}\right)^{-1 / 2} \mathrm{yr}$. In order for this to be substantially longer than the current observing time span of $1.1 \mathrm{yr}$, say $P_{3}>7 \mathrm{yr}$, one requires $M_{3}>0.007 M_{\odot}$.

The separation corresponds to an angular separation $\theta_{3}=$ $\left(1-f^{2}\right)^{1 / 2} a_{3} / d=0 .^{\prime} 08\left(f\left(1-f^{2}\right) M_{3} / 0.1 M_{\odot}\right)^{1 / 2}$ (note that $0 \leq$ $\left.\left(f\left(1-f^{2}\right)\right)^{1 / 2} \leq 0.62\right)$. A more massive third object might be visible in the HST images (if not a white dwarf); however, we found no evidence for a close-by object. The closest object is star B of Ferraro et al. (2001); with a mass of about a turn-off mass and a separation of $\sim 1$.' 4 , it is too far away to produce the required acceleration.

\subsection{X-ray luminosity}

An independent measure of the spin-down luminosity can be obtained from the X-ray luminosity, of $\sim 8 \times 10^{30} \mathrm{erg} \mathrm{s}^{-1}$ (Grindlay et al. 2001a). The fraction of the spin-down luminosity that is converted into X-rays is not understood theoretically, but one can use other MSPs to make an empirical calibration. From the data collected by Possenti et al. (2002; esp. Fig. 2a), we find that for the observed X-ray luminosity, a spin-down luminosity of about $10^{34.2 \pm 0.6} \mathrm{erg} \mathrm{s}^{-1}$ is expected. The best guess is about an order of magnitude lower than that inferred from the measured period and period derivative, and thus supports the suggestion that the intrinsic period derivative is lower than the measured one. We stress, however, that the estimate is very uncertain. Furthermore, while the MSP in M 28 follows the relation defined by MSPs in the field, the sources in 47 Tuc seem somewhat less X-ray bright than expected from that relation (Grindlay et al. 2002). Indeed, the latter define a different relation which, if extrapolated, fits the observed properties of PSR J1740-5340.

The situation is complicated further by the fact that the companion might contribute to the observed X-ray flux. For rapidly rotating, active stars in and out of binaries, the X-ray luminosity saturates at $\sim 0.1 \%$ of the bolometric luminosity (e.g., Stauffer et al. 1994; Patten \& Simon 1996; Dempsey et al. 1997). For the companion of PSR J1740-5340, $L_{\text {bol }} \simeq 2.0 L_{\odot}$, and hence its X-ray flux could be as high as $\sim 8 \times 10^{30} \mathrm{erg} \mathrm{s}^{-1}$, i.e., $100 \%$ of the flux observed. If the companion does contribute to the observed flux, this might also explain the observed variability (Grindlay et al. 2001a). Interestingly, comparing its relatively hard X-ray colour with BY Dra systems (which contain an active, rapidly rotating star) and other MSPs in NGC 6397 and 47 Tuc (Grindlay et al. 2001b, 2001a), it seems more consistent with the former. Indeed, as mentioned in Sect. 1, Taylor et al. (2001) had, when the identification with PSR J1740-5340 was not yet known, classified the companion as a BY Dra system.

\subsection{Ramifications}

D'Amico et al. (2001b) suggested that, as in the black-widow pulsars, the radio eclipses were due to mass loss driven by pulsar irradiation. Given the observed lack of heating of the companion, this seems rather implausible. Instead, we think that an intrinsic wind of the companion is responsible, as has been suggested for the very unenergetic eclipsing pulsar PSR B1718-19 in NGC 6342 (Wijers \& Paczynski 1993). Such a wind can be sufficiently strong because of the companion's rapid (co)rotation.

If the lack of heating is indeed due to an intrinsic spin-down rate lower than that observed, this affects the inferred values of the magnetic field strength and characteristic age of the pulsar. From our observations, we infer a spin-down luminosity a factor $>10$ smaller than inferred from the period derivative, implying that the intrinsic period derivative $\dot{P}_{\text {int }}>\dot{P}_{\text {obs }} / 10 \simeq$ $2 \times 10^{-20}$. Hence, the inferred magnetic field strength would become $<3 \times 10^{8} \mathrm{G}$ and the characteristic age $>3 \times 10^{9} \mathrm{yr}$.

\section{Current and evolutionary state}

Based on our analysis of HST photometry of PSR J1740-5340, we found that the companion has radius $R_{\text {comp }}=1.60 \pm 0.17 R_{\odot}$ and temperature of $T_{\text {eff }}=5410 \pm 50 \mathrm{~K}$, implying a luminosity $L_{\mathrm{bol}}=2.0 \pm 0.4 L_{\odot}$ (for the Gratton et al. distance, the radius and luminosity would be $1.52 \pm 0.08 R_{\odot}$ and $1.8 \pm$ $0.2 L_{\odot}$, respectively). From the ellipsoidal variations, we infer that the system's inclination is $>48^{\circ}$. For a pulsar mass in the range $1.2<M_{\mathrm{MSP}}<2.4 M_{\odot}$, this implies a companion mass in the range $0.14<M_{\text {comp }}<0.38 M_{\odot}$. In this range, the companion is just short of filling its Roche lobe (at just over the 2- $\sigma$ level, however, it may be filling its Roche lobe completely).

From our UVES spectrum, we find that the companion has low metallicity, as expected for membership of NGC 6397, that its radial velocity is consistent with membership for a mass ratio in the range $5<Q<7$, and that it rotates rapidly, 
$v_{\text {rot }} \sin i=52 \pm 4 \mathrm{~km} \mathrm{~s}^{-1}$, more or less consistent with it rotating synchronously with the orbit.

The largest surprise was that the light curve showed no evidence for heating. We discussed this at length and by an admittedly long chain of reasoning concluded that most likely the measured period derivative was dominated not by intrinsic spin-down, but by acceleration from a third object orbiting or passing by the system.

The picture that emerges is one of a system somewhat like an RS CVn binary, in which the co-rotating companion of the pulsar is an active star with a relatively strong but variable wind, which disperses and absorbs the pulsar signal for extended periods, in particular around superior conjunction of the pulsar. With this, we suggested an answer to the third of the three puzzles mentioned in the introduction, viz., the origin of the material causing the eclipses. We will now discuss the remaining two.

\subsection{Location in the cluster}

The location of the system is puzzling because it is far out of the core, at 0.55 or eleven core radii, even though the total mass of $\gtrsim 1.6 M_{\odot}$ is well above the turn-off mass of $0.8 M_{\odot}$. At the current position, the mass segregation time is rather short, between 1 and $10 \mathrm{Myr}$ depending on how close to the core the system is brought in its current orbit (Binney \& Tremaine 1987; Spitzer 1987; we used a system mass of $1.7 M_{\odot}$, current radius of $0.4 \mathrm{pc}$, enclosed mass of $2000 M_{\odot}$, and total number of objects in the cluster of $3 \times 10^{5}$ ). This suggests that the system was kicked out of the core, presumably by a close encounter. Probably, also, this interaction happened not too long ago, since it seems unlikely that the system was kicked out very far, and happens to be in the last stages of sinking back to the core at the present time. On the other hand, if the system really is young, one may wonder why not many more similar systems are known. Independent of the timescale, if there is indeed a third component in the current system, the interaction must have been between (at least) four components, the remaining object(s) to have left the core in the direction opposite to that of the pulsar triple system.

\subsection{Properties of the companion}

The companion of PSR J1740-5350 clearly is an unusual star: given its low mass, it should be on the main sequence, but it is far too large and bright for that. Might the star have been more massive originally? If so, did it lose its mass gradually, due to binary evolution, or impulsively, in an encounter? Alternatively, could the star have gotten bloated? We discuss these options briefly in turn.

\subsubsection{Binary evolution}

If the star originally was more massive, it could have lost its mass due to mass transfer in "regular" binary evolution. This has been suggested by Burderi et al. (2002) and Ergma \& Sarna (2002). Due to the mass transfer, the neutron star is expected to be spun up to millisecond periods. As the suggested solutions stand, there are some small problems: Ergma \& Sarna include heating by the pulsar, for which we see no evidence, and Burderi et al. end up with a slightly too massive companion, of $0.45 M_{\odot}$. We have little doubt, however, that suitable tuning could remedy this. Furthermore, in both models, the mass-transfer rate is only a few $10^{-10} M_{\odot} \mathrm{yr}^{-1}$, which implies a mass-transfer stage of several Gyr following its ejection from the core. Thus, one has to appeal to a coincidence that we happen to observe it in its last few Myr of sinking back.

A more pressing problem seems to be that in both models the companion is expected to continue to fill its Roche lobe. This is only marginally consistent with the fit to the light curve. Furthermore, it requires the assumption that, somehow, at some point the MSP switches on and manages to blow away all the matter thrown at it (which is a substantial amount in the model of Burderi et al. 2002). This would seem to require an energetic pulsar; however, we see no evidence for this radiation hitting the companion.

\subsubsection{A stripped turn-off star}

Could the encounter that kicked the system out of the core also have caused the companion to reach its present state? A possibility is that during a multiple-object interaction, the neutron star passed a star very closely, within about three stellar radii, which caused the star to be partially disrupted. Such a scenario was suggested by Zwitter (1993) for the eclipsing pulsar PSR B1718-19. In order for the disruption not to be complete, the star would have had to have a well-developed core, i.e., be a sub-giant or giant. During the interaction, some mass may been accreted by the neutron star. This could explain the $1 \mathrm{~s}$ period of PSR B1718-19, but would likely be insufficient to spin up a neutron star to the $3.65 \mathrm{~ms}$ period observed for PSR J1740-5340. If so, the neutron must have been a MSP already before the interaction.

It is not quite clear how a star reacts after rapidly losing more than half its mass, likely followed by a phase of strong tidal dissipation. Still, given that the luminosity for (sub-)giants depends mostly on the mass of the core, which would not change, one might suppose ending up with a star with roughly the same luminosity as it had before. For PSR J1740-5340, the luminosity of the companion is indeed comparable to the turnoff luminosity. Unfortunately, no prediction seems yet possible for the temperature or radius.

\subsubsection{A bloated main-sequence star}

For PSR B1718-19, the scenario invoking a stripped turnoff star cannot be correct, as the companion luminosity is far too low (Van Kerkwijk et al. 2000). Instead, the observations are consistent with another scenario, suggested by Wijers \& Paczynski (1993), viz., that the companion interacted with a neutron star in a less destructive manner, but was bloated during the subsequent circularisation phase. Could the same be true for PSR J1740-5340? We see two possible problems. First, since the orbit has been circularised, there are no energy sources any 
more in the companion except for nuclear processes. Hence, the companion should be shrinking on its thermal timescale, which is rather short, $G M^{2} / R L=1.0 \times 10^{6} \mathrm{yr}$.

The second problem is that, in contrast to what is the case for PSR B1718-19 (Verbunt 1994), the difference in total energy between the original eccentric orbit and the current circular one, $\left|E_{\text {circ }}\right|-\left|E_{\text {ecc }}\right|<\left|E_{\text {circ }}\right|=G M_{\mathrm{MSP}} M_{\text {comp }} / 2 a=$ $1.2 \times 10^{47} \mathrm{erg}$, is substantially smaller than the binding energy of $\frac{6}{7} G M^{2} / R=1.0 \times 10^{48}$ erg for a completely convective, $0.3 M_{\odot}, 0.3 R_{\odot}$ main-sequence star. This makes it doubtful that the star could be bloated sufficiently. Bloating of just the outer layers would not help: at the present luminosity, these would shrink back very rapidly.

\subsection{Sub-subgiants and red stragglers}

The combination of luminosity and temperature shown by the companion of PSR J1740-5350 is unusual, but not unique. Edmonds et al. (2002) pointed out the similarity with a number of objects found in 47 Tuc by Albrow et al. (2002). Albrow et al. dubbed these "red stragglers", and noted that their properties are similar to two so-called "sub-subgiants" in the old open cluster M 67, which are discussed in detail by Mathieu et al. (2002). All these objects are found well to the red of the main sequence, and about half a magnitude below the bottom of the red-giant branch. For all, the implied luminosities are comparable to those of turn-off stars, like for PSR J1740-5340.

Mathieu et al. (2002) note that the two M 67 sub-subgiants most likely are in or close to thermal equilibrium, since given the short thermal timescales it would otherwise be unlikely to find two in a (relatively) sparse cluster like M67. Albrow et al. (2002) suggest that for all sources the peculiar properties are due to mass transfer driven by evolution. This can indeed produce such stars (as shown also by the models for PSR J1740-5340 discussed above). For both objects in M 67, however, this is not possible: one is in an eccentric orbit, and the other is underfilling its Roche lobe (Mathieu et al. 2002).

Could these sub-subgiants result from a close encounter? Since the luminosities are so close to those of turn-off stars, it seems unlikely they are tidally bloated stars (as also suggested by the thermal timescale argument). In our list of scenarios for PSR J1740-5340 (which may of course be incomplete), this leaves only the possibility of stripped (sub-)giant. As mentioned, it is at present not clear whether such a stripping would lead to a star with the observed temperature, and to what extent the properties would depend on, e.g., the actual amount of mass loss.

The observed positions in the colour-magnitude diagram, close to the extension of the giant branch, might be taken to suggest the star has turned into a giant, but of lower mass than is possible by normal evolution. If so, since the lowest possible core masses for (sub-)giants are similar even among different (old) clusters, the similarity in their (starting) luminosities follows naturally. Also, since the further evolution would be on the slow nuclear timescale, finding relatively many would be possible. Finally, if this explanation holds, "red stragglers" would be an appropriate name: just like blue stragglers are not strange in their properties as such, but rather in their apparent youth (relative to what should be their contemporaries), the red stragglers would be strange in their apparent old age.

\section{Future work}

We have shown that it is possible to obtain reasonably good echelle spectra of the companion star in PSR J1740-5340. The next obvious step is to obtain several more spectra and measure the full radial velocity curve of the companion star. It should not be unduly difficult to obtain an accuracy of $K_{\text {comp }}$ well below $1 \mathrm{~km} \mathrm{~s}^{-1}$, which in turn will yield a mass ratio accurate at well below the $1 \%$ level. Apart from the double neutron star binaries, the mass ratio of PSR J1740-5340 would be the most accurately known mass ratio of a binary with a compact object.

Given that the mass ratio $Q$ can in principle be known extremely accurately, the only remaining quantity needed to obtain good component masses is the inclination $i$. In this case we are at a slight disadvantage compared to, e.g., studies of $\mathrm{X}$-ray binaries, since we cannot assume the companion fills its Roche lobe for the purposes of ellipsoidal modelling. As a result there is a large range of inclinations where the fits to the light curves are good (Fig. 5). We must use other constraints to narrow down this range, namely the radius of the companion from the $H S T$ photometry and the rotational velocity of the companion. Improvement of the former requires a better distance, which may already have become available with the detailed work of Gratton et al. (2002); for a very precise distance, one will have to wait for dedicated astrometric space missions. The precision of the measurement of the rotational velocity of the companion star, however, will improve with additional echelle spectra. From Fig. 5f, one sees that very high accuracy is required: even given a precisely known value of $Q$, an uncertainty in $v_{\text {rot }} \sin i$ of $1 \mathrm{~km} \mathrm{~s}^{-1}$ corresponds to a final uncertainty in the mass of $\sim 0.2 M_{\odot}$.

The allowed inclination range can also be reduced by using light curves with better phase coverage and higher statistical quality. Observations over several binary orbits should be obtained in order to verify that the light curves are indeed ellipsoidal, check that they are stable, and look for the presence of short-period variations such as those caused by star spots (if present, these could be used to verify the assumption of co-rotation). NGC 6397 has been well studied, and a large number of suitable observations no doubt exist in personal archives (e.g. Rubenstein \& Bailyn 1996; Kaluzny 1997), although likely advanced techniques such as image subtraction will be needed to recover good light curves.

It should be relatively easy to obtain an average echelle spectrum of the companion star with a signal-to-noise ratio on the order of 100 . This would allow one to do a detailed abundance analysis, and search for clues to the evolution of this system; e.g. a captured and perturbed low-mass main sequence companion will have a different composition than an initially higher-mass star that has lost a substantial amount of mass (e.g., Ergma \& Sarna 2002).

Finally, further radio timing, as well as detailed inspection of a good set of spectra, would allow one to search for evidence for the third star that we suggested was present. Higher 
signal-to-noise and time resolved X-ray observations might clarify whether the X-ray emission arises from the pulsar or from the companion.

While revising this article, we became aware of a preprint of Kaluzny et al. (2002) reporting ground-based photometry and low-resolution spectroscopy of the companion of PSR J1740-5340. They also find that the lightcurves, which have good phase coverage, are well described by ellipsoidal modulation. In detail, however, the results show small but significant differences from ours, mostly because their 2002 lightcurves turns out to have slightly smaller amplitude than our 1995 HST lightcurves. The main conclusions of our work, however, remain unchanged, in particular that there is no evidence for irradiation. Kaluzny et al. discuss the change in lightcurve amplitude and mention that it could reflect a change in inclination due to a third body, which we invoked above to explain the lack of irradiation. As said, this hypothesis can be tested with further timing. A more mundane and therefore perhaps better solution, also suggested by Kaluzny et al., is the presence of star spots. Finally, Kaluzny et al. measure radial velocities and derive a value for the radial-velocity amplitude, of $137.2 \pm 2.4 \mathrm{~km} \mathrm{~s}^{-1}$. This would imply $Q=5.15$. This value is consistent with the limits derived above, but significantly lower than our preliminary result based on further UVES observations, possibly because of blending with other stars in the badseeing conditions Kaluzny et al. were faced with.

Acknowledgements. We thank the staff at Paranal Observatory for performing the observations in a timely fashion, and the staff at ESO Garching for delivering the data to us in an expedited manner. This research made extensive use of ADS and SIMBAD. MHvK is supported by a fellowship of the Royal Netherlands Academy of Science.

\section{References}

Albrow, M. D., Gilliland, R. L., Brown, T. M., et al. 2001, ApJ, 559, 1060

Anthony-Twarog, B. J., \& Twarog, B. A. 2000, AJ, 120, 3111

Bell, J. F., Bailes, M., Manchester, R. N., Weisberg, J. M., \& Lyne, A. G. 1995, ApJ, 440, L81

Binney, J., \& Tremaine, S. 1987, Galactic Dynamics (Princeton: Princeton University Press)

Burderi, L., D'Antona, F., \& Burgay, M. 2002, ApJ, 574, 325

Callanan, P. J., van Paradijs, J., \& Rengelink, R. 1995, ApJ, 439, 928

Castilho, B. V., Pasquini, L., Allen, D. M., Barbuy, B., \& Molaro, P. 2000, A\&A, 361, 92

Claret, A. 2000, A\&A, 359, 289

D’Amico, N., Lyne, A. G., Manchester, R. N., et al. 2001a, ApJ, 548, L171

D’Amico, N., Possenti, A., Manchester, R. N., et al. 2001b, ApJ, 561, L89

D’Amico, N., Possenti, A., Fici, L., et al. 2002, ApJ, 570, L89

Dekker, H., D’Odorico, S., Kaufer, A., Delabre, B., \& Kotzlowski, H. 2000, SPIE, 4008, 534

Dempsey, R. C., Linsky, J. L., Fleming, T. A., \& Schmitt, J. H. M. M. 1997, ApJ, 478, 358

D'Odorico, S., Cristiani, S., Dekker, H., et al. 2000, SPIE, 4005, 121

Dolphin, A. 2000, PASP, 112, 1383

Edmonds, P. D., Gilliland, R. L., Heinke, C. O., Grindlay, J. E., \& Camilo, F. 2001, ApJ, 557, L57
Edmonds, P. D., Gilliland, R. L., Camilo, F., Heinke, C. O., \& Grindlay, J. E. 2002, ApJ, in press [astro-ph/0207426]

Ergma, E., \& Sarna, M. J. 2002, A\&A, submitted [astro-ph/0203433]

Ferraro, F. R., Possenti, A., D’Amico, N., \& Sabbi, E. 2001, ApJ, 561, L93

Ford, E. B., Joshi, K. J., Rasio, F. A., \& Zbarsky, B. 2000, ApJ, 528, 336

Gratton, R. G., Carretta, E., Bragaglia, A., Clementini, G., \& Grundahl, F. 2002, to appear in New Horizons in Globular Clusters Astronomy, ed. G. Piotto, G. Meylan, G. Djorgowski, \& M. Riello, PASP Conf. Ser.

Grindlay, J. E., Heinke, C. O., Edmonds, P. D., Murray, S. S., \& Cool, A. M. 2001a, ApJ, 563, L53

Grindlay, J. E., Heinke, C. O., Edmonds, P. D., \& Murray, S. S. 2001b, Science, 292, 2290

Grindlay, J. E., Heinke, C. O., Edmonds, P. D., \& Camilo, F. 2002, in Neutron Stars in Supernova Remnants, ed. P. O. Slane, \& B. M. Gaensler, ASP Conf. Ser., in press [astro-ph/0112484]

Hauschildt, P. H., Baron, E., \& Allard, F. 1997, ApJ, 525, 871

Harris, W. E. 1996, AJ, 112, 1487

Holtzman, J. A., Burrows, C. J., Casertano, S., et al. 1995, PASP, 107, 1065

Kaluzny, J. 1997, A\&AS, 122, 1

Kaluzny, J., Rucinski, S. M., \& Thompson, I. B. 2002, AJ, submitted [astro-ph/0209345]

Kulkarni, S. R., \& Hester, J. J. 1988, Nature, 335, 801

Kurucz, R. L. 1979, ApJS, 40, 1

Lattimer, J. M., \& Prakash, M. 2001, ApJ, 550, 426

Marsh, T. R., Robinson, E. L., \& Wood, J. H. 1994, MNRAS, 266, 137

Mathieu, R. D., van den Berg, M., Torres, G., et al. 2002, AJ, in press [astro-ph/0209568]

Montes, D., \& Martín, E. L. 1998, A\&AS, 128, 485

Orosz, J. A., \& Hauschildt, P. H. 2000, A\&A, 364, 265

Pasquini, L., Liu, Q., \& Pallavicini, R. 1994, A\&A, 287, 191

Patten, B. M., \& Simon, T. 1996, ApJS, 106, 489

Phinney, E. S. 1992, Phil. Trans. R. Soc. Lond. A, 341, 39

Possenti, A., Cerutti, R., Colpi, M., \& Mereghetti, S. 2002, A\&A, 387 , 993

Read, I. N. 1998, AJ, 115, 161

Reid, I. N., \& Gizis, J. E. 1998, AJ, 116, 2929

Rubenstein, E. P., \& Bailyn, C. D. 1996, AJ, 111, 260

Ryan, S. G., \& Deliyannis, C. P. 1998, ApJ, 500, 398

Spitzer, L. 1987, Dynamical Evolution of Globular Clusters (Princeton: Princeton University Press)

Stappers, B. W., van Kerkwijk, M. H., Bell, J. F., \& Kulkarni, S. R. 2001, ApJ, 548, L183

Stauffer, J. R., Caillault, J.-P., Gagne, M., Prosser, C. F., \& Hartmann, L. W. 1994, ApJS, 91, 625

Taylor, J. M., Grindlay, J. E., Edmonds, P. D., \& Cool, A. M. 2001, ApJ, 553, L169

Thévenin, F., Charbonnel, C., de Freitas Pacheco, J. A., et al. 2001, A\&A, 373, 905

Tonry, J., \& Davis, M. 1979, AJ, 841511

Trager, S. C., King, I. R., \& Djorgovski, S. 1995, AJ, 109, 218

Van Kerkwijk, M. H., Kaspi, V. M., Klemola, A. R., et al. 2000, ApJ 529,428

Verbunt, F. 1994, A\&A, 285, L21

Webbink, R. F. 1984, in Dynamics of Star Clusters, ed. J. Goodman, \& P. Hut (Dordrecht: Reidel), IAU Symp., 113, 541

Weisskopf, M. C., Hester, J. J., Tennant, A. F., et al. 2000, ApJ, 536, L81

Wijers, R. A. M. J., \& Paczynski, B. 1993, ApJ, 415, L115

Zwitter, T. 1993, MNRAS, 264, L3 